\title{
THE POTENTIAL FOR A HOMOGENEOUS CYLINDER IN A CYLINDRICAL COORDINATE SYSTEM
}

\author{
$\mathrm{BY}$ \\ WAN-XIAN WANG \\ Institute for Space Science and Technology, $1810 \mathrm{NW}$ 6th St., Gainesville, Florida
}

\begin{abstract}
Some special cases of the potential for a homogeneous cylinder in a cylindrical coordinate system may be treated by virtue of simple integrals, for example, the potential for a straight rod or wire segment and that for a homogeneous cylinder at the point on its axis. However, because of the involved mathematical operations, the analytical formula of the potential for a homogeneous cylinder at an arbitrary point has not been seen from others. In order to solve the problem, the author has taken the following steps: (1) expanding Green's function $e^{i k\left|\mathbf{r}^{\prime}-\mathbf{r}\right|} /\left|\mathbf{r}^{\prime}-\mathbf{r}\right|$ in the cylindrical coordinate system; (2) transforming Green's function $e^{i k\left|\mathbf{r}^{\prime}-\mathbf{r}\right|} /\left|\mathbf{r}^{\prime}-\mathbf{r}\right|$ into Green's function $1 /\left|\mathbf{r}^{\prime}-\mathbf{r}\right|$ by setting the wave number $k$ to be zero and integrating the separated azimuthal function $\cos ^{n}\left(\phi^{\prime}-\phi\right) ;(3)$ using the integral recursion relation for the function $r^{\prime 2 m+1} /\left[\left(z^{\prime}-z\right)^{2}+r^{\prime 2}+r^{2}\right]^{(4 m+1) / 2}$ with respect to $r^{\prime}$ and those for the functions $1 /\left[\left(z^{\prime}-z\right)^{2}+r^{2}\right]^{(2 m-1) / 2}$ and $1 /\left[\left(z^{\prime}-z\right)^{2}+r^{2}+a^{2}\right]^{(4 m-2 l-1) / 2}$ with respect to $z^{\prime}$, then we can complete the integrals for the function $1 /\left|\mathbf{r}^{\prime}-\mathbf{r}\right|$ and obtain the analytical expression of the potential for the cylinder in the cylindrical coordinate system. For numerical comparison, we have calculated the potentials for the cylinder and the prolate or oblate spheroid with equivalent volume and same high aspect ratio at far field point. The results are satisfactory.
\end{abstract}

1. Introduction. For simplicity and convenience, in the text we adopt the unit mass density of a cylinder and designate (see Fig. 1)

$$
\begin{aligned}
& z_{1}=z+h, \\
& z_{2}=z-h, \\
& r_{1}=\left(z_{1}^{2}+r^{2}\right)^{1 / 2}, \\
& r_{2}=\left(z_{2}^{2}+r^{2}\right)^{1 / 2}, \\
& r_{a}=\left(r^{2}+a^{2}\right)^{1 / 2}, \\
& \rho_{1}=\left(z_{1}^{2}+r_{a}^{2}\right)^{1 / 2},
\end{aligned}
$$

Received February 28, 1990. 


$$
\begin{aligned}
& \rho_{2}=\left(z_{2}^{2}+r_{a}^{2}\right)^{1 / 2}, \\
& Z=z^{\prime}-z, \\
& R_{0}=\left(Z^{2}+r^{2}\right)^{1 / 2}, \\
& R_{a}=\left(Z^{2}+r_{a}^{2}\right)^{1 / 2}, \\
& R=\left(Z^{2}+r^{\prime 2}+r^{2}\right)^{1 / 2}, \\
& H=-2 r^{\prime} r \cos \left(\phi^{\prime}-\phi\right),
\end{aligned}
$$

where $r^{\prime}, r, z^{\prime}, z$, and $\phi^{\prime}, \phi$ are the radial, axial, and azimuthal coordinates of the volume element of the cylinder and the field point, respectively, $a$ and $2 h$ are the radius and the length of the cylinder.

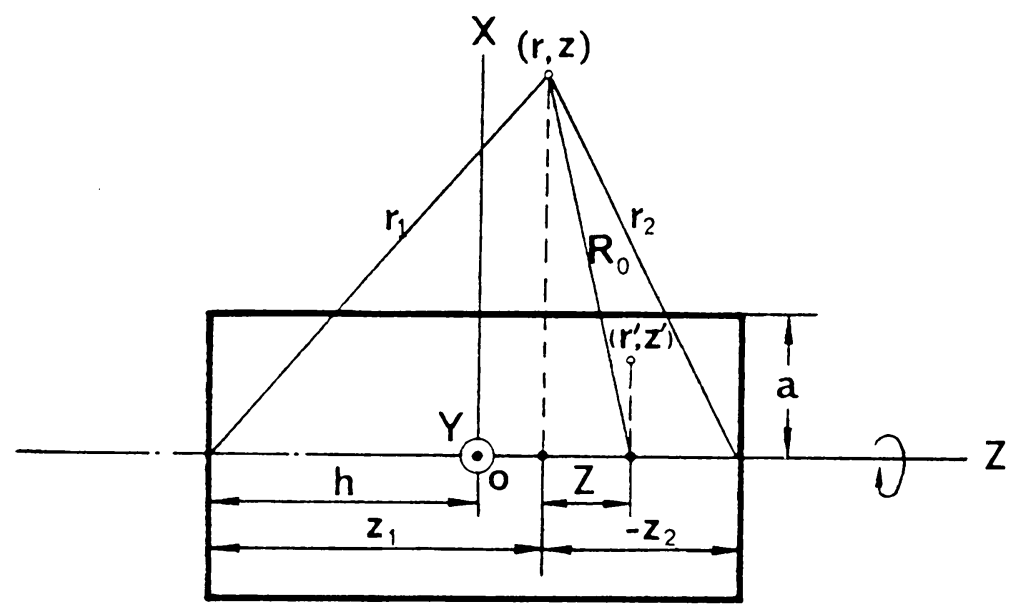

Fig. 1. A homogeneous cylinder. The radius is $a$, the half-length is $h$. The element of the cylinder is denoted by $\left(r^{\prime}, z^{\prime}\right)$, the field point by $(r, z)$.

The potential for a straight rod at the point $(r, z)$ in the rod-centered system is [1]

$$
V=\ln \left[\begin{array}{l}
z_{1}+r_{1} \\
z_{2}+r_{2}
\end{array}\right]=\ln \left[\begin{array}{l}
r_{1}+r_{2}+2 h \\
r_{1}+r_{2}-2 h
\end{array}\right] \text {. }
$$

The potential for a homogeneous cylinder at the point on its axis, $(0, z)$, in the cylindrical coordinate system is [2]

$$
V=\pi\left\{a^{2} \ln \left[\frac{z_{1}+\left(z_{1}^{2}+a^{2}\right)^{1 / 2}}{z_{2}+\left(z_{2}^{2}+a^{2}\right)^{1 / 2}}\right]+z_{1}\left(z_{1}^{2}+a^{2}\right)^{1 / 2}-z_{2}\left(z_{2}^{2}+a^{2}\right)^{1 / 2}+A\right\}
$$

in which the term $A$ is given by

$$
A=\left\{\begin{array}{lll}
-4 z h & \text { if } z>h & \text { (exterior point) } \\
-2 z^{2}-2 h^{2} & \text { if } h>z>-h & \text { (interior point) } \\
+4 z h & \text { if }-h>z & \text { (exterior point) }
\end{array}\right.
$$


In the cylindrical coordinate system, Green's function $1 /\left|\mathbf{r}^{\prime}-\mathbf{r}\right|$ may be written in the form

$$
1 /\left|\mathbf{r}^{\prime}-\mathbf{r}\right|=\left[\left(z^{\prime}-z\right)^{2}+r^{\prime 2}+r^{2}-2 r^{\prime} r \cos \left(\phi^{\prime}-\phi\right)\right]^{-1 / 2}=\left(R^{2}+H\right)^{-1 / 2},
$$

where $\mathbf{r}^{\prime}$ and $\mathbf{r}$ are the positional vectors of the volume element and the field point. It is much more difficult to expand Eq. (4) in the cylindrical coordinate system than the expression $\left[r^{\prime 2}+r^{2}-2 r^{\prime} r \cos \left(\phi^{\prime}-\phi\right)\right]^{-1 / 2}$ in the spherical coordinate system, the latter can be easily expanded as

$$
\left[r^{\prime 2}+r^{2}-2 r^{\prime} r \cos \left(\phi^{\prime}-\phi\right)\right]^{-1 / 2}= \begin{cases}\sum_{n=0}^{\infty} \frac{r^{\prime n}}{r^{n+1}} P_{n}\left(\cos \left(\phi^{\prime}-\phi\right)\right) & \text { if } r^{\prime}<r, \\ \sum_{n=0}^{\infty} \frac{r^{n}}{r^{n+1}} P_{n}\left(\cos \left(\phi^{\prime}-\phi\right)\right) & \text { if } r^{\prime}>r,\end{cases}
$$

where $r^{\prime}$ and $r$ are the radial distances of the volume element and the field point.

To expand Eq. (4), we will start from the expansion of $e^{i k\left|\mathbf{r}^{\prime}-\mathbf{r}\right|} /\left|\mathbf{r}^{\prime}-\mathbf{r}\right|$ in the cylindrical coordinate system and then set the wave number $k$ to be zero in Green's function and its expansion.

2. Expansion of Green's function $e^{i k}\left|\mathbf{r}^{\prime}-\mathbf{r}\right| /\left|\mathbf{r}^{\prime}-\mathbf{r}\right|$ in the cylindrical coordinate system [3]. From Lommel's expansion [4], we can separate the argument $\left(R^{2}+H\right)^{1 / 2}$ into the arguments $R$ and $H$ such that

$$
\begin{gathered}
{\left[k\left(R^{2}+H\right)^{1 / 2}\right]^{-1 / 2} J_{1 / 2}\left(k\left(R^{2}+H\right)^{1 / 2}\right)=\sum_{n=0}^{\infty} \frac{k^{2 n}(-H / 2)^{n}}{n !}(k R)^{-(n+1 / 2)} J_{n+1 / 2}(k R),} \\
{\left[k\left(R^{2}+H\right)^{1 / 2}\right]^{-1 / 2} J_{-1 / 2}\left(k\left(R^{2}+H\right)^{1 / 2}\right)=\sum_{n=0}^{\infty} \frac{k^{2 n}(H / 2)^{n}}{n !}(k R)^{-(n+1 / 2)} J_{-n-1 / 2}(k R),} \\
\text { for }|H|<R^{2}, \quad \text { (6.2) }
\end{gathered}
$$

wherein the Bessel functions $J_{n+1 / 2}(x)$ and $J_{-n-1 / 2}(x)$ can be converted to the spherical Bessel and Neumann functions of order $n: \sqrt{2 x / \pi} j_{n}(x)$ and $(-1)^{n+1}$ $\times \sqrt{2 x / \pi} n_{n}(x)$. The inequality required in Eq. (6.2) is in accordance with the following inequalities:

$$
R^{2}=\left(z^{\prime}-z\right)^{2}+r^{\prime 2}+r^{2} \geq r^{\prime 2}+r^{2} \geq 2 r^{\prime} r \geq\left|-2 r^{\prime} r \cos \left(\phi^{\prime}-\phi\right)\right|=|H|
$$

except the point with $z^{\prime}=z, r^{\prime}=r$, and $\phi^{\prime}-\phi=0$ or $\pi$, which is the removable singularity or ordinary point in the integral for $\cos ^{n}\left(\phi^{\prime}-\phi\right)$ which appears in the subsequent Eq. (7).

Further simplifications (similar to those used in [3]) for the combination of Eqs. (6.1) and (6.2) lead to

$$
\frac{e^{i k\left|\mathbf{r}^{\prime}-\mathbf{r}\right|}}{\left|\mathbf{r}^{\prime}-\mathbf{r}\right|}=i k \sum_{n=0}^{\infty} \frac{k^{2 n}\left(r^{\prime} r\right)^{n} h_{n}^{(1)}(k R)}{n !(k R)^{n}} \cos ^{n}\left(\phi^{\prime}-\phi\right),
$$

where $h_{n}^{(1)}$ is the spherical Hankel function of the first kind of order $n$. 
3. Green's function $1 /\left|\mathbf{r}^{\prime}-\mathbf{r}\right|$ and its integral with respect to $\left(\phi^{\prime}-\phi\right)$. Setting $k=0$ on both sides of Eq. (7) and using the form for $h_{n}^{(1)}(k R)$ at $k R=0$, we may obtain the expression

$$
\frac{1}{\left|\mathbf{r}^{\prime}-\mathbf{r}\right|}=\frac{1}{\sqrt{\pi}} \sum_{n=0}^{\infty} \frac{2^{n} \Gamma\left(n+\frac{1}{2}\right) r^{n}}{n !} \frac{r^{\prime n}}{R^{2 n+1}} \cos ^{n}\left(\phi^{\prime}-\phi\right),
$$

where the $\Gamma$-function is introduced.

The integral for $\cos ^{n}\left(\phi^{\prime}-\phi\right)$ with respect to $\left(\phi^{\prime}-\phi\right)$ shows

$$
\int_{0}^{2 \pi} \cos ^{n}\left(\phi^{\prime}-\phi\right) d\left(\phi^{\prime}-\phi\right)=\left\{\begin{array}{ll}
0 & \text { if } n=2 m+1, \\
2 \pi \frac{(2 m) !}{\left[2^{m} m !\right]^{2}} & \text { if } n=2 m .
\end{array} \quad m=0,1,2, \ldots\right.
$$

Hence, the potential for the cylinder may be expressed in the form of

$$
V=2 \sqrt{\pi} \sum_{m=0}^{\infty} \frac{\Gamma\left(2 m+\frac{1}{2}\right) r^{2 m}}{(m !)^{2}} \iint \frac{r^{\prime 2 m+1}}{R^{4 m+1}} d r^{\prime} d z^{\prime} .
$$

4. Integral for the function $r^{\prime 2 m+1} / R^{4 m+1}$ with respect to $r^{\prime}$. From the partial integral for the function $r^{2 m+1} / R^{4 m+1} \quad(m \neq 0)$, it can be proven that

$$
\begin{aligned}
\int_{0}^{a} r^{2 m+1} / R^{4 m+1} d r^{\prime}= & -\int_{0}^{a} \frac{r^{\prime 2 m}}{(4 m-1)} d\left(\frac{1}{R^{4 m-1}}\right) \\
= & -\frac{a^{2 m}}{(4 m-1)\left(R_{a}\right)^{4 m-1}} \\
& +\frac{2 m}{(4 m-1)} \int_{0}^{a} r^{\prime 2 m-1} / R^{4 m-1} d r^{\prime} ;
\end{aligned}
$$

from the recursion formula then we can deduce that

$$
\begin{aligned}
\int_{0}^{a} r^{\prime 2 m+1} / R^{4 m+1} d r^{\prime}= & -\sum_{l=0}^{m} \frac{2^{2 l+1}(4 m-2 l-2) !(2 m) ! m !}{(4 m) !(2 m-l-1) !(m-l) !}\left[\frac{a^{2 m-2 l}}{\left(R_{a}\right)^{4 m-2 l-1}}\right] \\
& +\frac{2^{2 m+1}(2 m) !(2 m-2) ! m !}{(4 m) !(m-1) !}\left[\frac{1}{\left(R_{0}\right)^{2 m-1}}\right] .
\end{aligned}
$$

If $m=0$, the integral for the function $r^{\prime} / R$ yields

$$
\int_{0}^{a} r^{\prime} / R d r^{\prime}=R_{a}-R_{0}
$$

Thus, Eq. (10) can be written as follows:

$$
\begin{aligned}
V=V_{0}+2 \pi \sum_{m=1}^{\infty} \frac{r^{2 m}}{m !} & {\left[\frac{(2 m-2) !}{2^{2 m-1}(m-1) !} \int_{-h}^{h} \frac{d z^{\prime}}{\left(R_{0}\right)^{2 m-1}}\right.} \\
& \left.-\sum_{l=0}^{m} \frac{(4 m-2 l-2) ! a^{2 m-2 l}}{2^{4 m-2 l-1}(2 m-l-1) !(m-l) !} \int_{-h}^{h} \frac{d z^{\prime}}{\left(R_{a}\right)^{4 m-2 l-1}}\right]
\end{aligned}
$$


and

$$
\begin{aligned}
V_{0} & =2 \pi \int_{-h}^{h}\left(R_{a}-R_{0}\right) d z^{\prime} \\
& =2 \pi \int_{-(z+h)}^{-(z-h)}\left(R_{a}-R_{0}\right) d Z \\
& =\pi\left\{r_{a}^{2} \ln \left[\frac{z_{1}+\rho_{1}}{z_{2}+\rho_{2}}\right]-r^{2} \ln \left[\frac{z_{1}+r_{1}}{z_{2}+r_{2}}\right]+z_{1}\left(\rho_{1}-r_{1}\right)-z_{2}\left(\rho_{2}-r_{2}\right)\right\} .
\end{aligned}
$$

5. Integrals for the functions $1 /\left(R_{0}\right)^{2 m-1}$ and $1 /\left(R_{a}\right)^{4 m-2 l-1}$ with respect to $z^{\prime}$. The expression in Eq. (14.1) is still integrable with respect to $z^{\prime}$. For convenience, we will discuss it in the two cases.

(1) $m=1$. The potential separated from the second part of Eq. (14.1) would be

$$
\begin{aligned}
V_{1} & =2 \pi r^{2}\left\{\frac{1}{2} \int_{-h}^{h} \frac{d z^{\prime}}{R_{0}}-\sum_{l=0}^{1} \frac{(2-2 l) ! a^{2-2 l}}{2^{3-2 l}[(1-l) !]^{2}} \int_{-h}^{h} \frac{d z^{\prime}}{\left(R_{a}\right)^{3-2 l}}\right\} \\
& =\pi r^{2}\left[\int_{-h}^{h} \frac{d z^{\prime}}{R_{0}}-\frac{1}{2} \int_{-h}^{h} \frac{a^{2} d z^{\prime}}{\left(R_{a}\right)^{3}}-\int_{-h}^{h} \frac{d z^{\prime}}{R_{a}}\right]
\end{aligned}
$$

and the resultant integrals are

$$
V_{1}=\pi r^{2}\left\{\ln \left[\frac{z_{1}+r_{1}}{z_{2}+r_{2}}\right]-\ln \left[\frac{z_{1}+\rho_{1}}{z_{2}+\rho_{2}}\right]-\frac{a^{2}}{2 r_{a}^{2}}\left(\frac{z_{1}}{\rho_{1}}-\frac{z_{2}}{\rho_{2}}\right)\right\} .
$$

(2) $m \neq 1$. We should investigate the integrals for the functions $1 /\left(R_{0}\right)^{2 m-1}$ and $1 /\left(R_{a}\right)^{4 m-2 l-1}$. Since the power indices of both functions are odd number, we just study the integral for the function $1 /\left(R_{a}\right)^{2 p+1}$.

Using variable transformation, the integral for $1 /\left(R_{a}\right)^{2 p+1}$ can be performed in such a way that

$$
\int_{-z_{1}}^{-z_{2}} \frac{d Z}{\left(\sqrt{Z^{2}+r^{2}+a^{2}}\right)^{2 p+1}}=\frac{1}{\left(r_{a}\right)^{2 p}} \int_{\alpha_{1}}^{\alpha_{2}} \cos ^{2 p-1} \alpha d \alpha,
$$

where $\alpha_{1}=\tan ^{-1}\left(-z_{1} / r_{a}\right)$ and $\alpha_{2}=\tan ^{-1}\left(-z_{2} / r_{a}\right)$. The integral recursion relation for $\cos ^{2 p-1} \alpha$ gives us

$$
\int_{\alpha_{1}}^{\alpha_{2}} \cos ^{2 p-1} \alpha d \alpha=\left.\sin \alpha \sum_{k=0}^{p-1} \frac{2^{2 k}[(p-1) !]^{2}(2 p-2 k-2) !}{[(p-k-1) !]^{2}(2 p-1) !} \cos ^{2 p-2 k-2} \alpha\right|_{\alpha_{1}} ^{\alpha_{2}},
$$

where $k$ is the summation index and is no longer the wave number.

With the aid of Eq. (18) and the substitutions of $\sin \alpha_{1}=-z_{1} / \rho_{1}, \sin \alpha_{2}=$ $-z_{2} / \rho_{2}, \cos \alpha_{1}=r_{a} / \rho_{1}, \cos \alpha_{2}=r_{a} / \rho_{2}, p=2 m-l-1$ in the integral for the function $1 /\left(R_{a}\right)^{4 m-2 l-1}$ and those of $\sin \alpha_{1}=-z_{1} / r_{1}, \sin \alpha_{2}=-z_{2} / r_{2}, \cos \alpha_{1}=$ $r / r_{1}, \cos \alpha_{2}=r / r_{2}, p=m-1$ in the integral for the function $1 /\left(R_{0}\right)^{2 m-1}$, we can 
finally arrive at

$$
\begin{aligned}
& V=V_{0}+V_{1} \\
&+2 \pi \sum_{m=2}^{\infty} \frac{1}{m !}\left\{\frac{(m-2) !}{2^{2 m-2}} \sum_{k=0}^{m-2} \frac{2^{2 k}(2 m-2 k-4) !}{[(m-k-2) !]^{2}}\right. \\
& \times\left(\frac{z_{1}}{r_{1}^{2 m-2 k-3}}-\frac{z_{2}}{r_{2}^{2 m-2 k-3}}\right)\left(r^{2 m-2 k-2}\right) \\
&-\sum_{l=0}^{m} \frac{(2 m-l-2) !}{2^{4 m-2 l-2}(m-l) !} \sum_{k=0}^{2 m-l-2} \frac{2^{2 k}(4 m-2 l-2 k-4) !}{[(2 m-l-k-2) !]^{2}} \\
&\left.\times\left(\frac{z_{1}}{\rho_{1}^{4 m-2 l-2 k-3}}-\frac{z_{2}}{\rho_{2}^{4 m-2 l-2 k-3}}\right)\left(\frac{r^{2 m} a^{2 m-2 l}}{r_{a}^{2 k+2}}\right)\right\}
\end{aligned}
$$

and

$$
V_{0}+V_{1}=\pi\left\{a^{2} \ln \left[\frac{z_{1}+\rho_{1}}{z_{2}+\rho_{2}}\right]+z_{1}\left(\rho_{1}-r_{1}\right)-z_{2}\left(\rho_{2}-r_{2}\right)-\frac{a^{2} r^{2}}{2 r_{a}^{2}}\left(\frac{z_{1}}{\rho_{1}}-\frac{z_{2}}{\rho_{2}}\right)\right\} .
$$

Eq. (19.1) reduces to the exact same form as Eq. (3) if $r=0$; at the center of the cylinder, the potential will be

$$
V=\pi\left\{a^{2} \ln \left[\frac{\left(h^{2}+a^{2}\right)^{1 / 2}+h}{\left(h^{2}+a^{2}\right)^{1 / 2}-h}\right]+2 h\left(h^{2}+a^{2}\right)^{1 / 2}-2 h^{2}\right\} .
$$

Therefore, Eqs. (19.1) and (19.2) are the general expressions of the potential for the cylinder in the cylindrical coordinate system.

By using Eqs. (19.1) and (19.2), it is also available to evaluate the potential for the cylinder at the point on its meridian, $(r, 0)$, as the traditional method is unable to deal with.

It is worth pointing out that Eqs. (19.1) and (19.2) will remain the same form at either interior or exterior point of the cylinder because of the parities of $r^{\prime}, r$ and $z^{\prime}, z$ in Eq. (8).

6. Numerical comparison and discussion. Since we do not have another analytic formula to calculate the potential for a homogeneous cylinder at an arbitrary point so as to make the comparison, the numerical results of the potentials for prolate and oblate spheroids with equivalent volume and same high aspect ratio of the cylinders (at high aspect ratio, the spheroid and the cylinder appear to be alike) have been used for this purpose.

For an elongated prolate spheroid, we assume that the semimajor axis $a_{s}=6.1$, the semiminor axis $b_{s}=1.1$, hence the semifocal distance $l=\left(a_{s}^{2}-b_{s}^{2}\right)^{1 / 2}=6.0$. The radius of the equivalent-volume calculated elongated cylinder, $a=b_{s} \sqrt[3]{\frac{2}{3}}$, and the half-length of the cylinder $h=a \times a_{s} / b_{s}$. The spheroidal radial and angular coordinates of the field point, $\xi$ and $\eta$, are taken as $\xi=\frac{113}{15}$ and $\eta=0.8$. Thus, the cylindrical coordinates of the point will be $r=l\left(\xi^{2}-1\right)^{1 / 2}\left(1-\eta^{2}\right)^{1 / 2}=26.88$ 
and $z=l \xi \eta=36.16$. The potential for the prolate spheroid at the point $(r, z)$, if the unit mass density is adopted, can be calculated by [5]

$$
V=\frac{4}{3} \pi a_{s} b_{s}^{2} / l \times\left[Q_{0}(\xi)-Q_{2}(\xi) P_{2}(\eta)\right]=0.687324684284 \ldots,
$$

where $Q_{0}(\xi)$ and $Q_{2}(\xi)$ are the Legendre functions of the second kind,

$$
Q_{0}(\xi)=\frac{1}{2} \ln \left[\frac{\xi+1}{\xi-1}\right] \quad \text { and } \quad Q_{2}(\xi)=Q_{0}(\xi) P_{2}(\xi)-\frac{3}{2} \xi
$$

The computation of the potential for the cylinder at the point $(r, z)$ quickly converges on 12 th place while the summation index $m \geq 3$ :

$$
V=0.687643849782 \ldots
$$

For a flattened oblate spheroid, we assume the same $a_{s}, b_{s}$, and $l$. The radius of equivalent-volume calculated disk, $a=a_{s} \sqrt[3]{\frac{2}{3}}$, and the half-length, $h=a \times b_{s} / a_{s}$. The spheroidal radial and angular coordinates of the field point are given by $\xi=$ $112 / 15$ and $\eta=0.8$. Therefore, $r=l\left(\xi^{2}+1\right)^{1 / 2}\left(1-\eta^{2}\right)^{1 / 2}=27.12$ and $z=l \xi \eta=$ 35.84. The potential for the oblate spheroid is

$$
V=i \frac{4}{3} \pi a_{s}^{2} b_{s} / l \times\left[Q_{0}(i \xi)-Q_{2}(i \xi) P_{2}(\eta)\right]=3.808547455474 \ldots,
$$

where

$$
Q_{0}(i \xi)=i\left[\tan ^{-1} \xi-\frac{\pi}{2}\right] \text { and } Q_{2}(i \xi)=Q_{0}(i \xi) P_{2}(i \xi)-\frac{3}{2}(i \xi) \text {. }
$$

The computation of the potential for the disk at the field point $(r, z)$ converges on 12th place while $m \geq 5$ :

$$
V=3.808902776405 \ldots
$$

The tiny differences after 4 th place between the potentials for the prolate or oblate spheroid and the cylinders at high aspect ratio in Eqs. (21) and (23) or Eqs. (24) and (26) are in the expectation in the view of their different geometrical shapes; certainly, their potentials at low aspect ratio would be quite different.

It has been shown in the numerical computations that the convergence for the expressions of Eqs. (19.1) and (19.2) upon the summation index $m$ at any field point $(r, z)$ is very fast. In the practical calculation of the potential for a homogeneous cylinder, it is enough to take the upper limit of $m$ being $2 \sim 3$ in order to keep the result accurate up to $4 \sim 5$ th place.

Figures 2, 3, and 4 show the contour plots of the homogeneous cylinders with radius $a=1.0$ and aspect ratio $2 h / a=1,2$, and 5 , respectively. The equipotential surfaces are as like as spheroidal, evolved from oblate ones to prolate ones. As is expected, all the equipotential surfaces become spherical at far field. For example, the values of potential remain same till 3 rd place on the sphere of radius $|\mathbf{r}|=15$ (where $|\mathbf{r}|=\left(r^{2}+z^{2}\right)^{1 / 2}$ ) in $2 h / a=1$ case, $|\mathbf{r}|=12$ for $2 h / a=2$, and $|\mathbf{r}|=24$ for $2 h / a=5$. 


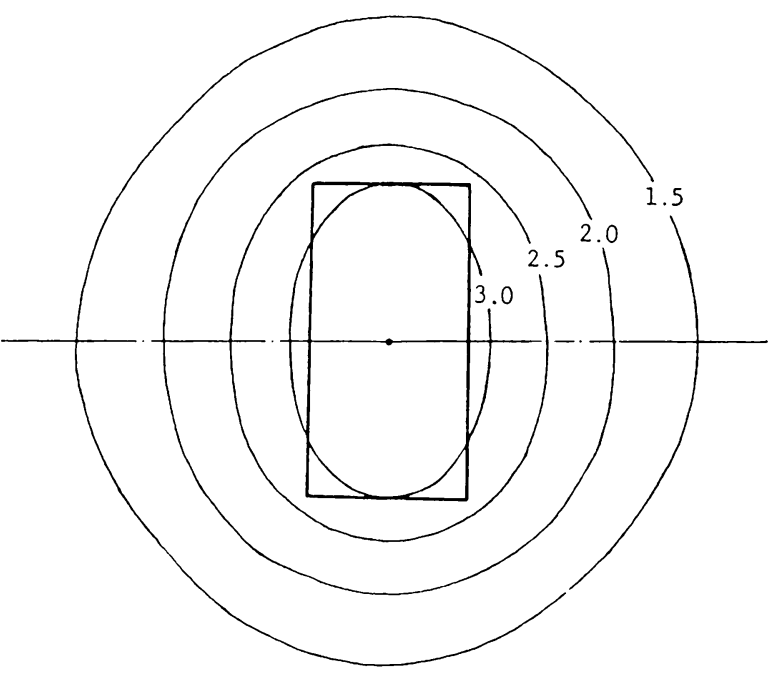

FIG. 2. Equipotential surfaces for the cylinder with aspect ratio $2 h / a=1$ and $a=1.0$.

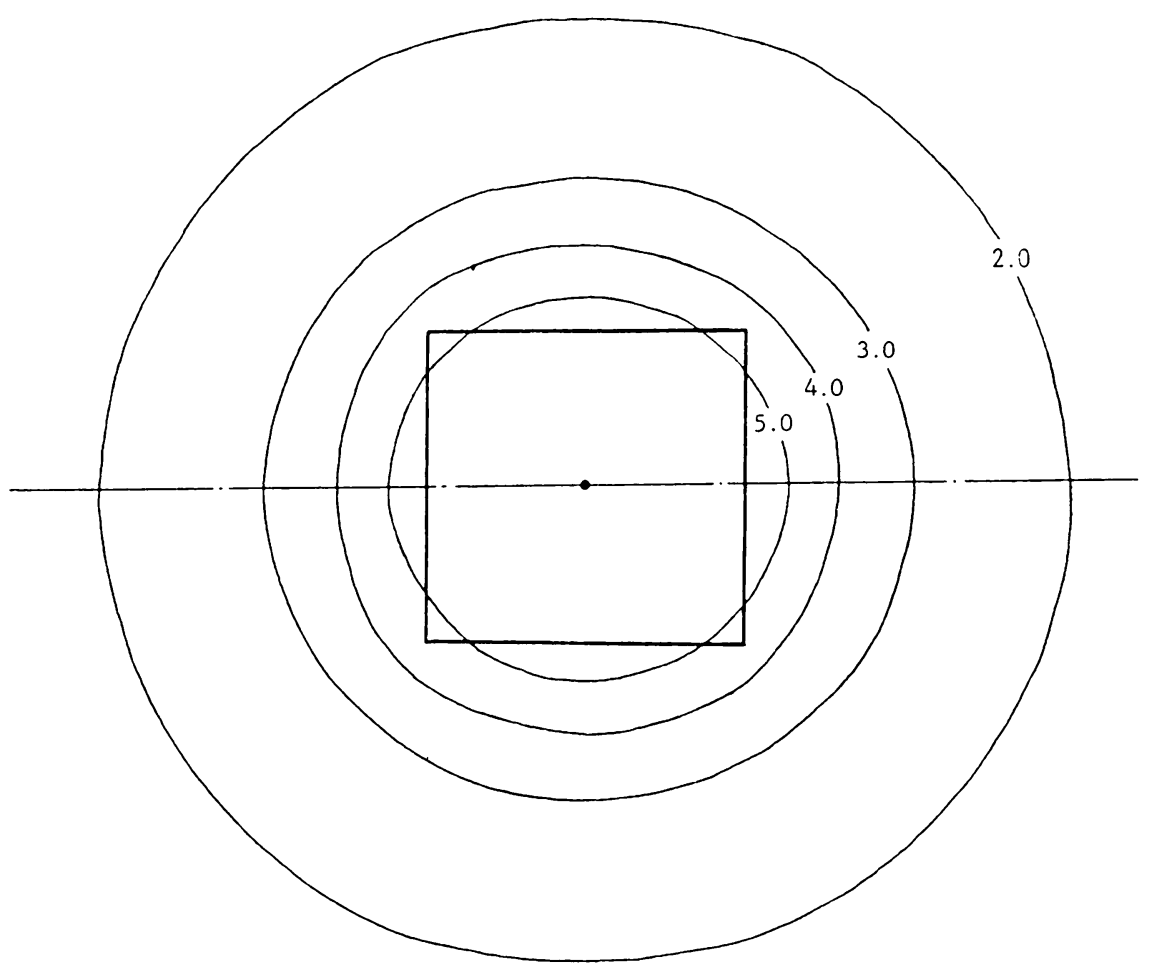

Fig. 3. Equipotential surfaces for the cylinder with aspect ratio $2 h / a=2$ and $a=1.0$. 


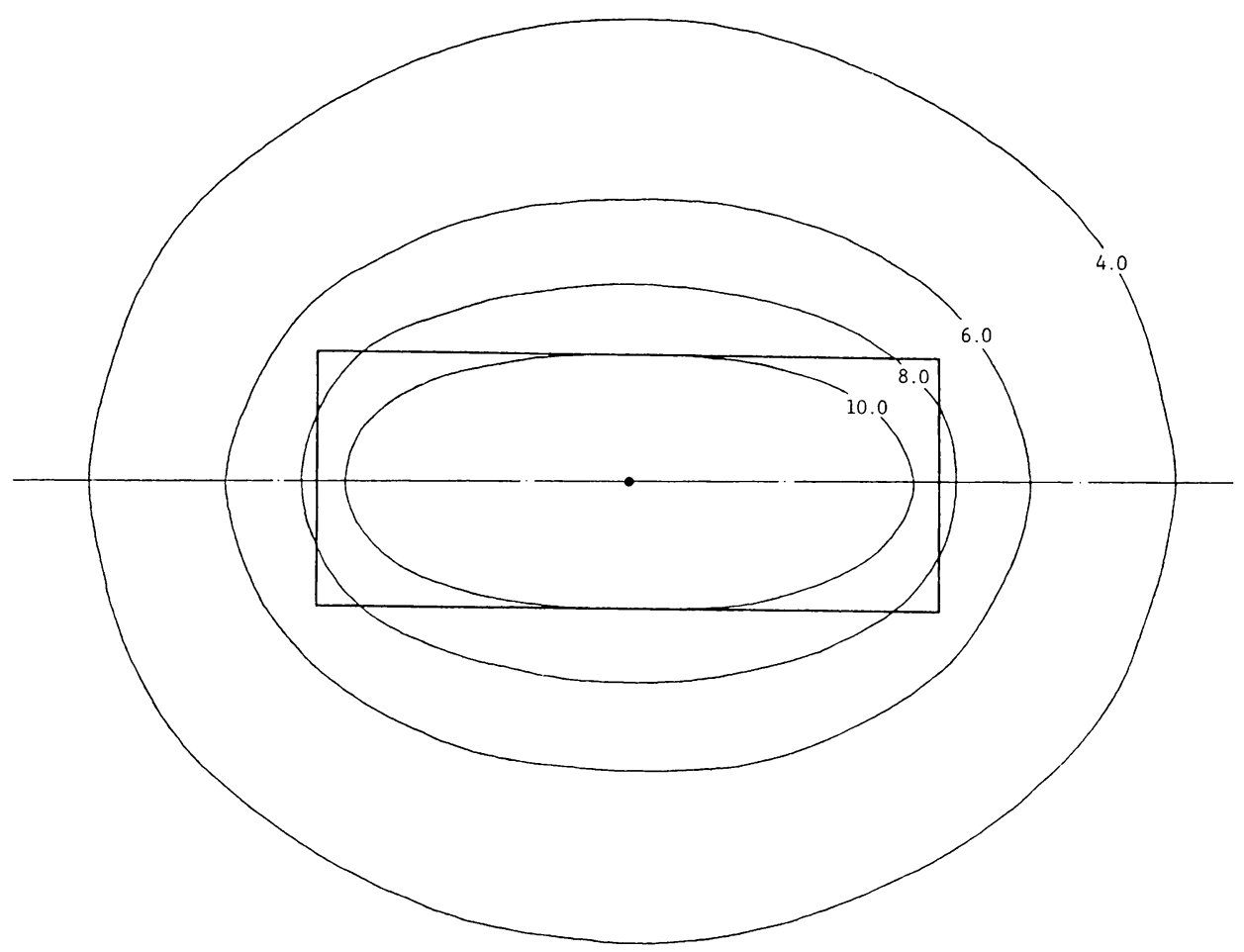

FIG. 4. Equipotential surfaces for the cylinder with aspect ratio $2 h / a=5$ and $a=1.0$.

Acknowledgment. The author thanks the referee for his careful verification on all the derivations and his valuable suggestion for contour plots.

\section{REFERENCES}

[1] O. D. Kellogg, Foundations of Potential Theory, Dover, New York, 1929

[2] W. D. MacMillan, The Theory of the Potential, Dover, New York, 1958

[3] W. X. Wang, Expansion of the Green's function in a cylindrical coordinate system, Quart. Appl. Math. 48, 499-501 (1990)

[4] G. N. Watson, A Treatise on the Theory of Bessel Functions, Cambridge University Press 1962

[5] T. M. MacRobert, Spherical Harmonics, Pergamon Press, 1927 